\title{
Algunas notas para el estudio del exilio en la obra poética de Cristina Peri Rossi
}

\author{
Alejandra $\mathrm{M}^{\mathrm{a}}$ Aventín FontANA \\ Departamento de Humanidades: Filosofía, Lenguaje y Literatura \\ Universidad Carlos III de Madrid \\ aaventin@hum.uc3m.es
}

\section{RESUMEN}

El presente artículo aspira a ser una introducción a la reflexión y el estudio del exilio histórico en la obra poética de la escritora uruguaya Cristina Peri Rossi (Montevideo, 1941) quien, tras abandonar su país natal en 1973, no regresó a él tras el fin del régimen dictatorial en 1985. El artículo consta de una primera parte en la que se reflexiona sobre el exilio como proceso vital, marcado por la escisión que se genera en el individuo y las consecuencias que éste tiene, al tiempo que se incluyen algunas declaraciones de la escritora que hemos considerado importantes para poder evaluar la repercusión que el exilio tuvo en su escritura poética. La segunda parte se centra en rastrear algunas de las claves del imaginario poético de Peri Rossi en su producción previa al momento de la partida para valorar cómo el exilio histórico repercute en su evolución. Finalmente, la tercera y última parte está dedicada al análisis de Estado de exilio, primer poemario redactado tras su partida y que, por expreso deseo de la autora, no fue publicado hasta el año 2003. Se trata de una obra clave que nos permite reflexionar sobre el impacto del exilio histórico en su voz poética. Su análisis ha sido realizado tomando como eje vertebral de la lectura las etapas señaladas por los estudiosos de la migración y el exilio Leon y Rebeca Grinberg como características del proceso que el sujeto experimenta.

Palabras clave: exilio, margen, transgresión, escisión.

An introduction to the study of exile in Cristina Peri Rossi's poetry

\begin{abstract}
This text is an introduction to the study of historical exile in the Uruguayan Cristina Peri Rossi's poetry work. Peri Rossi (Montevideo, 1941) had to leave her homeland for political reasons in 1973 and though in 1985 the didactorship ended, she never returned to Uruguay. The article is divided in three parts. The first one is focused on making a reflection about exile, which is approached and thought as a vital process signed by the split generated in the
\end{abstract}


individual with a clear emphasis on the particular case of Cristina Peri Rossi. The second part shows the results of the search for key aspects of Peri Rossi's poetry work previous to exile in order to evaluate the impact of such an event in the evolution of her writing. The third and final part of the text is dedicated to the analysis of Estado de exilio, which was the first book of poems written by the author after exile, though it was not published until 2003. Estado de exilio is a very important book to understand the effect of historical exile in Cristina Peri Rossi's literary voice. In order to address its reading, we have used the works of Leon and Rebeca Grinberg about migration and exile with a particular attention to the three periods that, attending to their studies, the exiled goes through.

Key words: exile, margin, transgression, split.

\title{
1. COORDENADAS GENERALES DE LA TRAVESÍA
}

A los veintinueve años de edad, Cristina Peri Rossi (Montevideo, 1941) abandonaba su Uruguay natal en el barco que hacía regularmente la línea Montevideo-Buenos Aires-Génova rumbo a Barcelona el 4 de octubre de 1972, como medida preventiva ante la represión existente en la capital uruguaya, producida por la convulsa situación política que vivía Uruguay durante aquel año. Era la primera vez que la escritora salía de su país. En el libro de poemas titulado Estado de exilio -que la autora escribió recién llegada a la ciudad condal en 1973 y que por cuestiones de pudor no vio la luz hasta el año 2003 (Peri Rossi 2005: 13)- encontramos una composición titulada "Viaje" en la que Peri Rossi nos proporciona una emotiva crónica de éste:

\author{
Mi primer viaje \\ fue el del exilio \\ quince días de mar \\ sin parar $[\ldots]$ \\ Quince días de agua \\ sin luces de neón \\ sin calles sin aceras sin ciudades [...] \\ Quince días de mar \\ e incertidumbre $[. .$. \\ Nadie te despidió en el puerto de partida \\ nadie te esperaba en el puerto de llegada. (Peri Rossi 2005: 320-329)
}

Poco tiempo después, Peri Rossi era testigo de la instauración de un régimen dictatorial que frustraba su deseo de volver a tierras uruguayas por mucho tiempo. Pese a su lucha inicial y clandestina desde Barcelona para derrocar la dictadura, la democracia no llegaría hasta 1985. Al dolor por la repentina partida se sumaría poco tiempo después la prohibición de su obra literaria en Uruguay.

En 1993, Cristina Peri Rossi ya había establecido su residencia permanente en Barcelona y hablaba así del exilio, que al igual que otros muchos ciudadanos hispanoamericanos, cambió su vida para siempre: "El exilio fue una experiencia larga, dolorosa, totalizadora, que no cambiaría por ninguna otra" (Peri Rossi 1993). 
En su obra Psicoanálisis de la migración y del exilio, Leon y Rebeca Grinberg señalan la puesta en riesgo de la identidad y su posible fragmentación como consecuencia inmediata que tiene el exilio en el individuo, ya que supone la pérdida de los objetos más significativos y valorados: "personas, cosas, lugares, idioma, cultura, costumbres, clima", etc. Tras la marcha se inicia un proceso de duelo que se vive como "un cambio catastrófico en la medida en que ciertas estructuras se transforman en otras a través de momentos de dolor, desorganización y frustración". Sin embargo, "estos momentos una vez superados y elaborados, darán la posibilidad de un verdadero crecimiento y evolución enriquecida de la personalidad" (Grinberg 1984: 102) al tiempo que si el sujeto cuenta "con capacidad de elaboración suficiente, no sólo superará la crisis, sino que, además ésta tendrá una cualidad de "renacimiento", (Grinberg 1984: 27). A la luz de estas consideraciones, podríamos concluir que las palabras pronunciadas por Peri Rossi podrían muy bien indicar que aparentemente la uruguaya ya habría superado entonces la aludida crisis propia del exilio a la que se referían los Grinberg.

El exilio consiste por lo tanto en una experiencia desgarradora que se inscribe dentro de un proceso psicológico que guarda una serie de pautas comunes para todos aquellos que lo han vivido. No obstante, el estudioso Francisco Caudet (2005: 185) señala que "las opiniones y matices acerca de la vivencia personal del exilio, varían de acuerdo con las circunstancias, el momento y las causas". Tal y como apunta la reflexión de los Grinberg, el exilio es un proceso; y tal y como explica el profesor Caudet se trata de una vivencia. El concepto de vivencia que la RAE define como "hecho de experimentar algo, y su contenido" subraya el carácter personal y único del exilio en cada individuo, mientras que el vocablo proceso nos remite a los aspectos comunes y generales de éste.

El presente texto aspira a ser una introducción al estudio del exilio histórico en la obra poética de Cristina Peri Rossi. Cabe señalar que en esta ocasión centraremos una parte importante del mismo en el ya citado libro de poemas Estado de exilio por considerarlo representativo, así como en su producción poética previa, en la que quedan esbozados algunos aspectos clave de su imaginario poético y en la que nos referimos igualmente a otros tipos de exilio presentes ${ }^{1}$.

\section{LA TRANSGRESIÓN COMO ACTO DE LIBERTAD: LA OBRA POÉTICA DE CRISTINA PERI ROSSI ANTERIOR A LA PARTIDA}

Cristina Peri Rossi, poeta, ensayista y excepcional narradora, publicó su primer libro de poemas titulado Evohé en 1971, tras el notable éxito cosechado con los cuatro títulos de narrativa que lo precedieron: Viviendo (1963), Los museos abandona-

${ }^{1}$ Nos gustaría señalar que la obra poética de Cristina Peri Rossi consta de catorce libros hasta la fecha: Evohé (1971), Descripción de un naufragio (1975), Estado de exilio (escrito en 1973 pero publicado en 2003), Diáspora (1976), Lingüística general (1979), Europa después de la lluvia (1987), Babel bárbara (1991), Otra vez Eros (1994), Aquella noche (1996), Inmovilidad de los barcos (1997), Las musas inquietantes (1999), Estrategias del deseo (1999), Habitación de hotel (2007) y Playstation (2009). 
dos (1969), El libro de mis primos (1969) e Indicios pánicos (1970). El poemario causó un gran revuelo en el Montevideo de aquella época por su alta temperatura erótica, su claro talante subversivo y un homoerotismo entrevisto que tenía como objetivo, tal y como ha señalado Hugo J. Verani, "la búsqueda de un nuevo lenguaje" que comprometiera "al ser entero y la cristalización de una nueva conciencia poética en el mundo actual" (Verani 1995: 12).

El erotismo que encontramos en Evohé es un erotismo humanizador que aspiraba a "la necesidad de naturalizar las fuerzas de los instintos" como una parte integrante del amor y del deseo concebido como pulsión esencial de la vida. Éste encuentra su más claro antecedente en la poesía uruguaya en la voz de la gran poeta modernista Delmira Agustini. La ironía y el sarcasmo, la naturalidad y la ternura conviven en unos versos que, puestos en boca de una mujer en una sociedad patriarcal en los años 70, muy pronto se convertirán en objeto de ataque para quienes no vieron con buenos ojos que aquello que la sociedad había restringido al ámbito privado trascendiera al público.

La escritura poética de Peri Rossi se convierte así en un exponente de que, tal y como señala Susana Reisz, "escribir como una mujer es una opción política" (Reisz 1996: 51). Pero de igual forma evidencia el exilio cultural que han sufrido las mujeres a lo largo de la historia, tema que la crítica académica ha desarrollado en títulos como Women's writing in exile (Broe e Ingram 1989) o Exilios femeninos (Cuder 1997) y al que también se refiere Parizad Tamara Dejbord en su obra Cristina Peri Rossi: escritora del exilio (Dejbord 1998: 41). Afirma Dejbord: "La mujer, como el exiliado, es un ser híbrido, que pertenece y no pertenece, a la vez, a culturas, a discursos y a mundos simultáneos" (Dejbord 1988: 45). Podemos decir por lo tanto que la escritura de la uruguaya refleja y denuncia, ya en un momento previo a su exilio histórico, otro tipo de exilio, el exilio femenino, con energía y determinación. Este hecho resulta importante ya que da muestras del talante pragmático de Cristina Peri Rossi en el período anterior a su marcha forzosa que mucho tendría que ver con cómo la uruguaya supo gestionar posteriormente el proceso de superación del exilio que la llevaría fuera de su país natal, transformando el duelo inicial en un camino provechoso para el autoconocimiento. Su obra poética se convierte así en un interesante territorio para reflexionar sobre la vivencia del exilio y sus consecuencias en la fragua del imaginario poético.

Edward Said explica que el escritor exiliado articula su discurso desde otro sitio, desde otro lugar en el que el mundo es sentido como territorio extranjero (Said 2000: 186), por lo que podríamos concebir este discurso como uno que nace desde el margen. Sin embargo queremos subrayar que, antes de ser desterrada de su país, Peri Rossi ya articulaba su discurso desde el margen en un doble sentido: en primer lugar como mujer, pero igualmente como ciudadana comprometida que aspiraba a denunciar y a contribuir al cambio de la situación política y social de un país que vivía una profunda crisis. Dice la propia Peri Rossi: "escribo contra la realidad. Empecé a hacerlo porque la realidad que veía a mi alrededor -en mi casa, primero; luego en mi país- no me gustaba. Y sigo escribiendo, me parece, por la misma razón" (Dejbord 1998: 61). Esta labor queda reflejada en su obra narrativa de este período y es plasmada en el ámbito de lo poético en su siguiente libro de poemas que, como si 
de una premonición se tratara, lleva por título Descripción de un naufragio y que no publicaría hasta 1975 ya en Barcelona. No obstante, nótese que el poemario fue redactado en un momento anterior a su partida. De esta manera, el talante transgresor que ha sido a menudo apuntado como una de las características de las obras escritas desde el exilio por la crítica ${ }^{2}$, es ya entonces una dinámica habitual en la obra poética de Peri Rossi. Verani se refiere a él como una constante en los versos de la uruguaya: "la transgresión como acto de libertad hace maleable el mundo, en ello reside la clave de la aventura poética de Cristina Peri Rossi" (Verani 1995: 20).

Descripción de un naufragio propone, desde el tono conversacional (Narváez 1991: 24), adentrarnos en el mundo marítimo en el que la autora sitúa y rinde homenaje a todos aquellos que tuvieron que abandonar el país por motivos políticos y a los que desaparecieron, víctimas y náufragos de la defensa de unos ideales. El libro se abre con una composición titulada "Dedicatoria", el único poema de la obra que lleva título. En él podemos leer:

A todos aquellos navegantes

argonautas de un país en ruinas

desaparecidos en diversas travesías,

varias, que un día emprendieron navegaciones

de inciertos desenlaces. (Peri Rossi 2000: 107)

En sus versos, la fe se tambalea ante la furia de un mar que no es sino metáfora del dolor que brinda el fracaso, pero también, en otros momentos, oleaje suave que acuna recuerdos y que aviva el deseo de denuncia de los pasajeros, entre los que Peri Rossi se incluye y desde la cubierta del barco imaginario explica:

No fue nuestra culpa si nacimos en tiempos de penuria.

Tiempos de echarse al mar y navegar.

Zarpar en los barcos y remolinos

huir de guerras y tiranos

al péndulo

a la oscilación del mar. (Peri Rossi 2005: 121)

Pero el mar es también telón de fondo de la soledad y espacio regido por lo elemental y lo primigenio, ámbito apto para el reencuentro con el amor restaurador de una posible futura armonía:

\footnotetext{
${ }^{2}$ Para Julia Kristeva, el exilio es una forma de disidencia en tanto que el hecho de desplazarse conlleva la ruptura de los lazos anteriores (Kristeva 1986). Por su parte, Michael Ugarte en su obra Literatura española en el exilio. Un esudio comparativo se refiere a la transgresión propia de los autores exiliados y pone el ejemplo de dos autores españoles, si bien esta reflexión es fácilmente extensible al resto: "El exilio implica siempre algún tipo de conflicto. Los escritores desterrados están reñidos con el orden existente, ya sea político, literario o ambos. [...] Para algunos, como Cernuda y Goytisolo, transgredir se convirtió en la fuerza que impulsaba la mayoría de sus creaciones literarias al alabar su condición de exiliados. Por consiguiente, atravesar las barreras políticas conduce irremediablemente a atravesar otras barreras -las normas literarias, la moral convencional" (Ugarte 1999: 229).
} 


\author{
El mar. \\ El temor a la inmensidad \\ El arte de navegar \\ La facultad de amar \\ La soledad. (Peri Rossi 2005: 121)
}

La presencia de lo acuático vinculado a la esperanza de un renacer simbólico de la humanidad comparte su condición liberadora nuevamente con la presencia de la mujer y ese erotismo humanizador que concilia contrarios y emparenta con las fuerzas cósmicas (Narváez 1991: 25), permitiendo la restauración de la unidad quebrada.

Descripción de un naufragio nos da cuenta de la travesía y el naufragio de aquellos que, como Peri Rossi, quisieron un rumbo diferente para la historia de su país, y de la solidaridad y unidad colectiva con la que la escritora uruguaya vivió el desmembramiento definitivo anterior al destierro, cuando la situación de su Uruguay natal se había hecho insostenible y su vida, como la de otros muchos uruguayos, corría peligro de ser silenciada. Peri Rossi no pensaba entonces en abandonar Uruguay: "me costó mucho irme. La decisión de salvar el pellejo fue muy difícil. No quería irme y me costó mucho ponerme de acuerdo con esa decisión. Incluso después, durante mucho tiempo, me sentí muy culpable de haberme ido" (Dejbord 1998: 221). Esto convierte los versos de Descripción de un naufragio en portadores de un tercer tipo de exilio: el exilio interno o exilio interior, término que ha sido acuñado por la crítica para referirse a la situación de marginación que sufrieron todos aquellos que permanecieron dentro del territorio uruguayo durante esa época y que fue inicialmente acuñado por Paul Illie en su obra Literature and Inner Exile. Authoritarian Spain, 1939-1975 (1980: 11).

\title{
3. ESTADO DE EXILIO O LAS GEOGRAFÍAS DE LA MEMORIA
}

Diáspora (1976), poemario por el que Peri Rossi obtuvo el premio "Inventarios Provisionales" fue ya redactado en España y resultó la siguiente obra que la uruguaya publicó. Sin embargo, anterior a ésta, Peri Rossi ya había redactado su libro de poemas Estado de exilio que escribió como si de una catarsis necesaria se tratara. Dice su autora:

Las pérdidas, el desarraigo del exilio tienen siempre una fantasía de castración, de silencio. Mientras lloraba pensando que fuera de mi ciudad y de mi país [...], del semanario Marcha, del malecón con tamarindos de Piriápolis, del café de Sorocabana, lejos de mis alumnos y alumnas no iba a poder escribir una sola línea más en la vida, escribí de un tirón, en Barcelona, los poemas de Estado de exilio. Fue en 1973. No hice ningún esfuerzo por publicarlos [...] pensaba que esos poemas, nacidos de un dolor colectivo, iban a acentuar la sensación de desarraigo, de desgracias, de tragedia. (Peri Rossi 2005: 13-14)

Entre las varias acepciones que encontramos de la palabra estado en la RAE queremos destacar la siguiente: "situación en que se encuentra alguien o algo, y en espe- 
cial cada uno de sus sucesivos modos de ser o estar". Estado de exilio nos muestra exactamente ese intento de Cristina Peri Rossi por afrontar el exilio y relatar su situación: su amago por estar sin dejar de ser y su insistencia por ser sin dejar de estar y que en la obra gravitan alrededor del sentimiento de escisión que conlleva la partida. Leemos en el primer poema del libro: "Tengo un dolor aquí, / al lado de la patria" (Peri Rossi 2005: 287). Dolor y escisión aparecen reflejados en esta primera composición en la que, de un lado queda la patria, y del otro queda el "aquí,, el nuevo espacio al que la poeta sólo es capaz de referirse con el deíctico de lugar. El dolor con el que queda vertida la nueva geografía gravita a lo largo de toda la obra $\mathrm{y}$ resulta materializado en diferentes aspectos.

En primer lugar, cabe destacar que la poeta alterna el uso de la primera persona del singular, 'yo', con el de otras personas gramaticales en su intento por restituir la raíz cercenada y ser capaz de articularse para poder superar la catástrofe del destierro y la consiguiente desorganización interior. Peri Rossi emplea el 'nosotros' en un intento de permanecer vinculada al cuerpo social desgajado tras la partida y, en particular, a aquellos que como ella sufren la condición de ser exiliados. En "Exiliados II" leemos:

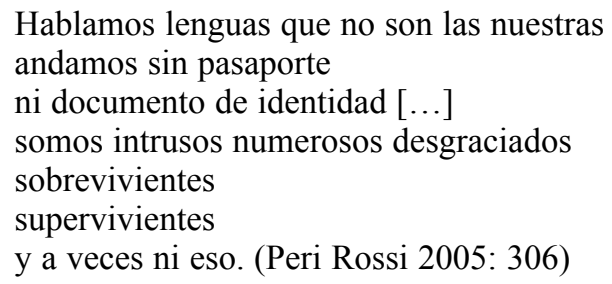

Asimismo encontramos el uso de la tercera persona del plural, que la uruguaya utiliza en un intento por distanciarse para hacer más efectiva la denuncia de esta situación y tomar distancia con una realidad y un estado difícil de aceptar. Dice Peri Rossi:

Algunos se han dejado crecer barba, otros, se han cortado la barba hay quien se pierde caminando por no poder dormir $[\ldots]$ Se reconocen por el acento, y por la tristeza de la mirada. (Peri Rossi 2005: 318)

En "Carta de mamá" la poeta plasma entrecomillada la voz de la progenitora desde la distancia: "Carta de mamá: 'Y si todos se van, hija mía, / ¿qué vamos a hacer los que nos quedamos?'” (Peri Rossi 2005: 289).

En la composición que coincide con el título del libro, "Estado de exilio", nos encontramos con un poema en el que el caos emanado del dolor aniquila la linealidad del discurso de la poeta que, inmóvil, es sólo capaz de "mirar diferente" (Peri Rossi 2005: 299). Los deícticos espaciales, temporales, así como los adverbios, se entremezclan para dar muestra de un ser que lucha por emplazarse y buscar en el exterior el calor humano que le sacuda de la soledad castradora: 
[...] muy pronto tan lejos bastante mal

siempre

dificultad palabras furiosa largo

extraño extranjero que más el árbol

sólo miro diferente

todo

fuera más humano. (Peri Rossi 2005: 299)

Previamente a esta composición destaca el segundo poema del libro en el que, en un momento anterior a la cuasi petrificación generada por su estado de exilio, Peri Rossi, incapaz de aceptar la realidad, sueña con su partida. Sin embargo, la confusión y el miedo paralizador no desaparecen:

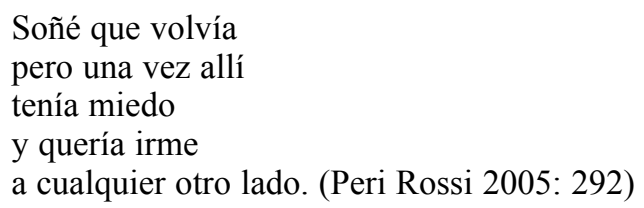

La búsqueda de nuevos referentes que calmen la ausencia de aquellos que le proporcionaba la geografía cotidiana, y a los que se refiere a lo largo de todo el libro, es otra constante que nos permite dar cuenta del aludido proceso de duelo y consiguiente reorganización interna a la que se refieren los Grinberg como característicos de este primer momento del exilio. En este sentido vemos una evolución. Si bien en un principio éstos son evocados como única realidad posible desde la nostalgia:

Una casa

un cuadro

una silla

[...] perdidos

pesan tanto como la ausencia de mamá. (Peri Rossi 2005: 297)

En "Geografia" son sustituidos por otros nuevos en un tono que denota un deseo por garantizar el éxito del proceso:

Los nuevos objetos triviales, perecederos, son mi mapa mi nueva geografía. (Peri Rossi 2005: 332)

Sin embargo, nótese que Peri Rossi se refiere a objetos o referentes perecederos, lo cual acentúa la idea de transitoriedad y provisionalidad que señala Francisco Caudet como frecuente en la escritura del exiliado (Caudet 2005: 23). Sin embargo, la ironía a la que la autora nos tiene acostumbrados no desaparece y actúa como estrategia discursiva para calmar la trágica verticalidad del sentimiento articulado en el discurso, tal como vemos en el poema "Cabina telefónica 1975":

[...] el exilio es tener un franco en el bolsillo y que el teléfono se trague la moneda $[\ldots]$ 
en el exacto momento en que nos damos cuenta

de que la cabina no funciona. (Peri Rossi 2005: 312)

En el ya citado Psicoanálisis de la migración y del exilio, Leon y Rebeca Grinberg distinguen tres etapas en el proceso que experimenta el exiliado: una primera etapa en la que prima el sentimiento de escisión, el dolor por todo lo abandonado y lo perdido, la soledad y el desamparo; una segunda etapa que está marcada por el sentimiento de la nostalgia y la pena del mundo perdido; y una tercera etapa caracterizada por la apertura hacia el nuevo entorno, la recuperación del deseo y de la capacidad de proyectar planes de futuro en el que el pasado ya no es vivido como el paraíso perdido (Grinberg 1984: 119).

Tal y como hemos podido constatar en nuestra aproximación a Estado de exilio, la obra da inevitablemente cuenta de la primera etapa marcada por esa escisión y el dolor que nace de ésta y que en el poema "El viaje" se concreta en los versos que cierran la composición: "Partir / es siempre partirse en dos" (Peri Rossi 2005: 329). Sin embargo, también nos muestra que la fragmentación vivida conduce a la poeta a un camino de aceptación de sus nuevas circunstancias en las que no parece haber hueco para el erotismo al que nos tiene acostumbrados pero sí lo hay para el deseo de volver a amar, tal y como se constata en el poema "XVI":

[...] es seguro que nuestra venganza será el amor

poder amar, todavía

poder amar, a pesar de todo. (Peri Rossi 2005: 311)

Este amor y el tono desafiante de la citada composición nos muestran el camino recorrido en Estado de exilio: éste nace de la geografía de la memoria, alterada por la nostalgia y la pérdida, aspectos que forman parte de la segunda etapa del proceso del exilio (Grinberg 1984: 119) y extiende sus baldosas a la promesa de volver a desear aprehender el mundo, actitud clave que es indicadora de la correcta marcha de la adaptación al nuevo estado y rasgo de la tercera etapa a la que se refieren los Grinberg (Grinberg 1984: 119). En el poema "Barnanit" que Peri Rossi incluye en el poemario, pese a ser escrito en un momento posterior, por considerarlo un puente entre Estado de exilio y Estrategias del deseo, poemario este último que cierra Poesía reunida; el entorno es espacio obligatorio, pero también es cuna y abrazo, reino de Eros victorioso y resurrecto, final de la travesía; y la lengua es espacio, patria íntima, desde la que reinventarse y conjugar el verbo amar:

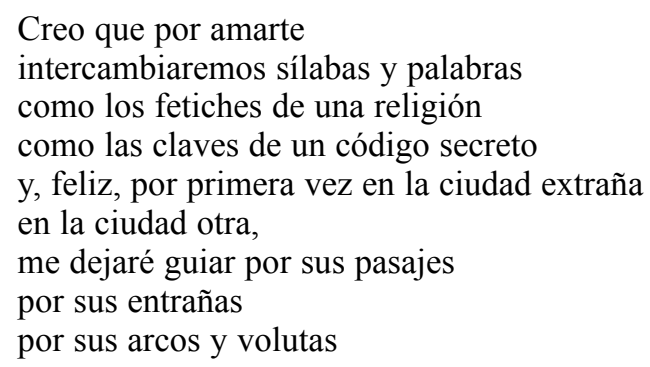


como la viajera por la selva en el medio del camino de nuestra vida. Las ciudades sólo se conocen por amor y las lenguas son todas amadas. (Peri Rossi 2005: 346)

\section{BIBLIOGRAFÍA}

BROE, Mary Lynn; e INGRAM, Angela (eds.) (1989): Women's writing in exile. Chapel Hill: The University of North Carolina Press.

CAUDET, Francisco (2005): El exilio republicano de 1939. Madrid: Cátedra.

CUDER, Pilar (ed.) (1999): Exilios femeninos. Huelva: Universidad de Huelva.

DEJBORD, Tamara Parizad (1998): Cristina Peri Rossi: escritora de exilio. Argentina: Galerna.

GRINBERG, Leon y Rebeca (1984): Psicoanálisis de la migración y del exilio. Madrid: Alianza.

ILLIE, Paul (1980): Literature and Inner Exile. Authoritarian Spain, 1939-1975. Baltimore: Johns Hopkins University Press.

KRISTEVA, Julia (1986): “A New Type of Intellectual: The Dissident”, in Moi, Toril (ed.), The Kristeva Reader. New York: Columbia University Press.

NARVAEZ, Carlos Raúl (1991): La escritura plural e infinita. El libro de mis primos de Cristina Peri Rossi. València: Albatros.

PERI ROSSI, Cristina (1993): Cristina Peri Rossi. Málaga: Centro Cultural de la Generación del 27.

- (2005): Poesía completa. Barcelona: Lumen.

REISZ, Susana (1996): Voces sexuadas. Género y poesía en Hispanoamérica. Lleida: Universitat de Lleida / Asociación Española de Estudios Literarios Hispanoamericanos.

SAID, Edward (2000): Reflections on Exile and Other Essays. Cambridge / Massachusets: Harvard University Press.

UGARTE, Michael (1999): Literatura española en el exilio. Un estudio comparativo. Madrid: Siglo XXI.

VERANI, Hugo J. (1995): "La rebelión del cuerpo y del lenguaje”, en Cosse, Romulo (ed.), Cristina Peri Rossi. Papeles críticos. Uruguay: Linardi y Risso. 